\title{
Parâmetros Psicométricos:
}

\section{uma Análise de Testes Psicológicos Comercializados no Brasil}

\section{Psychometrics Parameters: an analysis of the psychological tests commercialized in Brazil}

Resumo: As técnicas utilizadas na avaliação psicológica têm provocado questionamentos nas comunidades científicas e profissionais brasileiras, tanto no que se refere à qualidade dos instrumentos, de maneira geral, ao uso que os psicólogos fazem dos instrumentos, bem como em relação à validade geral dos resultados da avaliação psicológica. O presente trabalho teve como objetivo identificar as principais informações psicométricas a respeito dos instrumentos psicológicos presentes em seus manuais, tais como editora, data de publicação, variável medida, padronização, validade e precisão. Foram consultados 146 instrumentos de avaliação comercializados no Brasil por onze editoras. Os resultados indicaram que somente 28,8\% desses instrumentos relatam estudos de precisão, validade e padronização. Esses resultados sugerem que a Psicologia brasileira tem-se caracterizado por despender pouca atenção aos fundamentos científicos de sua prática profissional.

\section{Ana Paula Porto}

Noronha

Doutora em Psicologia: Ciência e Profissão pela Pontifícia Universidade Católica de Campinas. Docente do Curso de Psicologia e do Programa de Pósgraduação Stricto Sensu em Psicologia da Universidade São Francisco.

\section{Ricardo Primi}

Doutor em Psicologia pela Universidade de São Paulo. Docente do Curso de Psicologia e do Programa de Pósgraduação Stricto Sensu em Psicologia da Universidade São Francisco.

Coordenador do Laboratório de Avaliação Psicológica e Educacional (LabAPE).

João Carlos Alchieri

Doutor em Psicologia do Desenvolvimento pela Universidade Federal do Rio Grande do Sul. Docente da Universidade Federal do Rio Grande do Norte Palavras-Chave: Instrumentos psicológicos, avaliação psicológica, validade, precisão.

Abstract: Psychological assessment instruments have been questioned by the scientific and professional community in respect to their general psychometric quality, the use that psychologists give to their instruments and also to the general validity of the results of the psychological assessment. The aim of this paper is to identify the main psychometric information of the commercialized psychological tests as reported in their manuals, as well as publisher, date, construct measuring, standardization, validity and reliability. It analyzes 146 instruments commercialized by 11 editors. The results show that only $28.8 \%$ of these instruments report reliability, validity and standardization studies in their manuals. These results suggest that Brazilian Psychology has been characterized by a less than desirable concern about the scientific basis of its professional practices.

Key Words: Psychological tests, psychological assessment, validity, reliability.

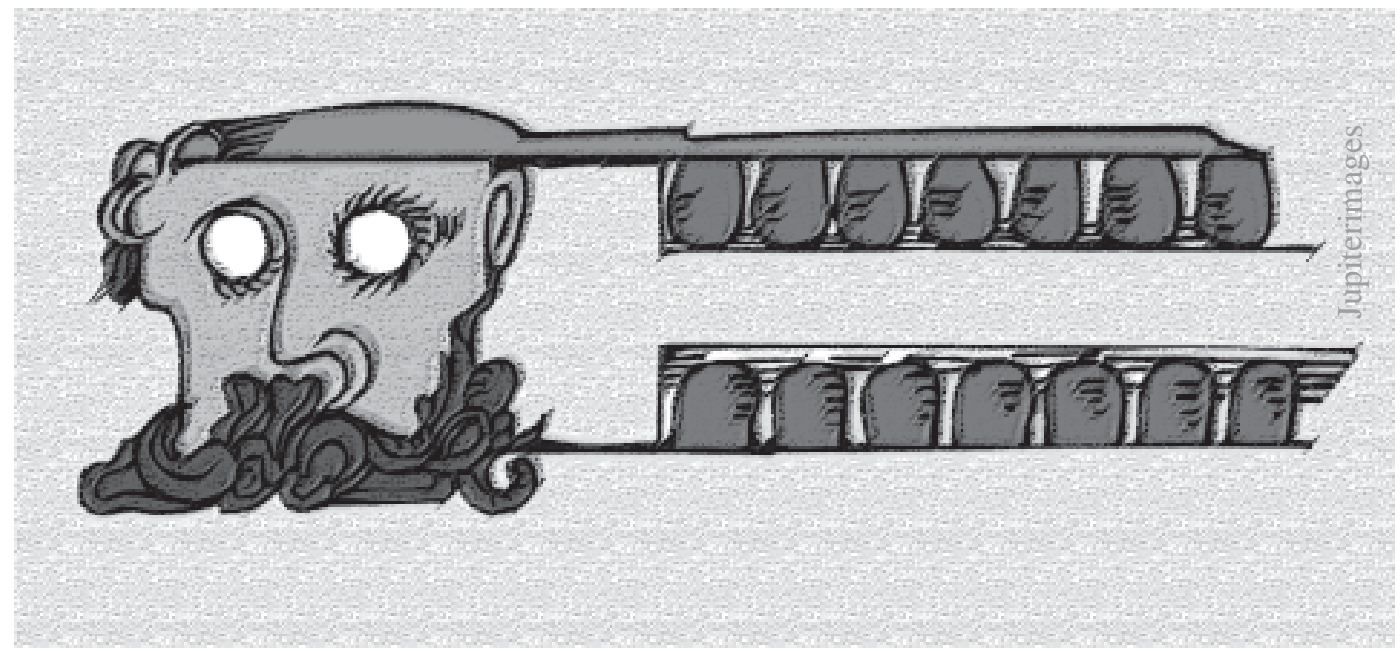

As técnicas utilizadas na avaliação psicológica têm provocado questionamentos nas comunidades científicas e profissionais brasileiras, tanto no que se refere à qualidade dos instrumentos, de maneira geral, ao uso que os psicólogos fazem dos instrumentos bem como em relação à validade geral dos resultados da avaliação psicológica.
Tais questionamentos já provocaram, e ainda hoje provocam, modificações na postura do psicólogo, que ora oscila em direção à idealização, ora ao menosprezo dos recursos que dispõe nessa área. A idéia do uso de instrumentos, originária na década de 30, de uma perspectiva técnica e pragmática da instrumentalização em Psicologia, caracterizava a ação do profissional como mais bem amparada no contexto cultural de uma ciência do comportamento humano. Assim sendo, o desenvolvimento de instrumentos ganhava um forte impulso para a aplicabilidade do entendimento 
psicológico nas mais diversas áreas de atuação. Posteriormente, nas décadas de 60 e 70 , os instrumentos foram criticados e o seu uso minimizado na atuação do profissional de Psicologia; dentre os motivos, encontra-se o advento do pensamento humanista, o movimento de contra-cultura, o questionamento a respeito das idéias tecnicistas e, principalmente, no Brasil, a associação dos modelos de avaliação com a cultura técnica norte-americana. Esses fatos históricos já foram amplamente discutidos por vários autores, dentre eles: Anastasi e Urbina (2000), Ancona-Lopez, (1987), Erthal (1987), Pasquali (1999) e Pasquali e Alchieri (2001).

Atualmente, os questionamentos apresentados pela sociedade frente aos resultados de avaliações psicológicas em seleção de pessoal, psicodiagnóstico, na obtenção de carteira de habilitação, entre outros contextos e possibilidades profissionais, levam a pensar e ponderar quanto aos diversos aspectos envolvidos na práxis do profissional de avaliação psicológica (Alves Filho, 2002).

Sem dúvida alguma, a avaliação psicológica, hoje, voltou a ter sua importância e seu status na atuação do psicólogo, tanto no que se refere às perspectivas internacionais quanto nas nacionais. Em recente relatório da Comissão de Testes do Colégio Oficial de Psicólogos (COP) e da Comissão Européia sobre Testes da Federação Européia de Associações Profissionais de Psicólogos (EFPPA), foram apontadas diretrizes internacionais para o estabelecimento de políticas para o uso dos testes psicológicos. Cabe destacar o papel delegado aos destinatários, ou seja, a responsabilidade de promover a escolha adequada dos instrumentos a serem utilizados bem como a preocupação acerca da sua qualidade e atualização (ITC, 2001). Não se questiona mais a utilidade dessa prática privativa do psicólogo, que, de alguma forma, representa e difunde a Psicologia enquanto ciência na sociedade. Ainda nessa perspectiva dos padrões de excelência para os instrumentos, recentemente, foi publicada uma revisão dos Standards for Educational and Psychological Tests (American Educational Research Association, American Psychological Association \& National Council on Measurement in Education, 1999), uma referência clássica quanto aos parâmetros dos instrumentos utilizados na avaliação psicológica.

No Brasil, a emergência, hoje, está centrada na discussão da qualidade e da elaboração de instrumentos nacionais de avaliação psicológica. A temática da qualidade dos instrumentos psicológicos é, atualmente, merecedora de investigações por parte de pesquisadores em diferentes regiões do País, e, dentre os estudos desenvolvidos, encontram-se aqueles que versam sobre o ensino e formação, sobre exercício profissional, sobre novas tecnologias e teorias que embasam a construção e validação de testes.

No que se refere aos estudos que discutem o ensino da avaliação psicológica, destaque deve ser dado aos trabalhos de Ribeiro (1996); Pereira e Carellos (1995); Jacquemin (1995); Kroeff (1988); Alves, Alchieri e Marques (2001) e Vasconcelos e Toledo de Santana (2001), Alchieri e Bandeira (2002). Embora tenham enfoques metodológicos diferentes, os trabalhos convergem para a necessidade de se repensar a formação profissional de forma que ela se adapte melhor às necessidades da prática do profissional.

Mais especificamente no que se refere ao uso dos instrumentos de avaliação, Azevedo, Almeida, Pasquali e Veiga (1996) constataram, no estudo realizado com psicólogos com o objetivo de conhecer os instrumentos mais utilizados e de saber a sua opinião a respeito dos testes, que metade da amostra acredita no valor dos testes, com a seguinte ressalva: que sejam usados de acordo com princípios éticos e em conjunto com outros instrumentos de avaliação.

Um dos primeiros levantamentos nacionais sobre o uso de testes psicológicos é o estudo de Hutz e Bandeira (1993), que aponta, em suas conclusões, além da discrepância entre a quantidade de instrumentos nacionais e internacionais, a falta de referências sobre os instrumentos mais utilizados no País. Os autores caracterizaram descritivamente tanto os testes objetivos quanto os projetivos, proporção de trabalho entre os mais utilizados em cada área e a ênfase quanto à necessidade de política de atualização do instrumental brasileiro.

Vários outros estudos têm dado destaque à qualidade dos instrumentos de avaliação psicológica, na tentativa de se resgatar a sua legitimidade (Prieto \& Muñiz, 2000; Noronha, 2001; Noronha, Sartori, Freitas \& Ottati, 2001; Noronha, Sartori, Freitas \& Ottati, 2002). Outros estudos versam sobre temas distintos, tais como as implicações relativas à revisão dos testes psicológicos (Silverstein, Marshall \& Nelson, 2000) e sobre a complexidade teórica da revisão de alguns instrumentos, como, por exemplo, os projetivos (Butcher, 2000; Link, 1999).

O Conselho Federal de Psicologia (CFP), ao longo das duas últimas décadas, criou espaços de interlocução com a sociedade científica na busca de soluções para a melhor da qualidade dos serviços relacionados à avaliação psicológica. Em 1980, foi criada a Comissão Nacional sobre Testes Psicológicos, objetivando discutir uma política
O Conselho Federal de Psicologia (CFP), ao longo das duas últimas décadas, criou espaços de interlocução com a sociedade científica na busca de soluções para a melhor da qualidade dos serviços relacionados à avaliação psicológica. 
quanto à área. Em 1986, houve uma segunda edição dessa comissão, que incluía algumas recomendações quanto ao uso de instrumentos psicológicos. Em 1997, surgiu a Câmara Interinstitucional de Avaliação Psicológica, decorrente do veto presidencial à avaliação psicológica do trânsito, a qual assessorou o CFP (2000) quanto à criação do Manual para Avaliação Psicológica de candidatos à Carteira Nacional de Habilitação e condutores de veículos automotores. Simultaneamente, foram realizadas discussões em fóruns regionais e nacionais de avaliação psicológica a fim de obter e encaminhar propostas sobre a formação e prática profissional na área. Em decorrência disso, surgiram, em 2001, duas resoluções regulamentando a ação profissional no que se refere aos laudos e aos instrumentos de avaliação psicológica (CFP, 2001a, 2001b).

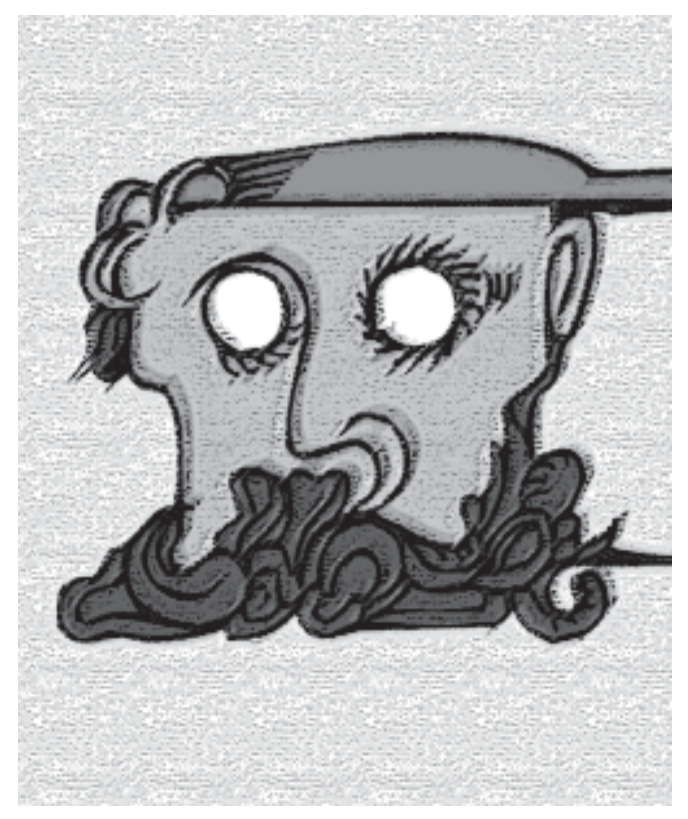

1 Nomeadamente, o ITC Guidelines on Adapting Tests, ITC Guidelines on Test Use da International Test Comission (ITC), o Standards for Educational and Psychological Testing publicado pela American Educational Research Association, American Psychological Association, National Council on Measurement in Education e o Guidelines for Educational and Psychological Testing " publicado pela Canadian Psychological Association.

2 Título traduzido do russo por Paulo Bezerra (in Vigotski, 2001).

A resolução CFP 02/2003 estabelece que os instrumentos de avaliação psicológica, no Brasil, para serem considerados como tal e estarem em condições de uso profissional, devem atender a um conjunto de requisitos mínimos estabelecidos pela Psicometria. A resolução apóia-se em documentos internacionais que tratam detalhadamente desse assunto ${ }^{1}$. Basicamente, esses requisitos indicam que os instrumentos devem possuir: (a) uma fundamentação teórica, (b) evidências empíricas de validade e precisão das interpretações propostas, (c) um sistema de correção e interpretação dos escores, (d) uma
Paralelamente ao movimento do CFP, na reunião da Associação Nacional de Pesquisa e PósGraduação em Psicologia (ANPPEP), em 2000, o grupo de trabalho em avaliação psicológica discutiu a sistematização do processo de avaliação da qualidade dos instrumentos comercializados. Desse evento, surgiu um grupo de pesquisadores dos Laboratórios de Instrumentos de Avaliação Psicológica (LIAP, UNISINOS) e Laboratório de Avaliação Psicológica e Educacional (LabAPE, USF) com o objetivo de (a) elaborar uma base de dados de instrumentos nacionais comercializados de avaliação psicológica, (b) identificar e caracterizar as principais informações a respeito dos instrumentos psicológicos (autor, tradutor, data de publicação, editora, atualizações e revisões), (c) descrever os principais indicadores técnicos dos instrumentos psicológicos, como: validade, precisão, padronização e (d) caracterizar os instrumentos psicológicos mais utilizados pelos profissionais de Psicologia em diversos contextos (Noronha, Alchieri \& Primi, 2001).

Dada a extensão da proposta, o presente artigo teve a intenção de apresentar um dos objetivos acima relacionados, ou seja, identificar e caracterizar as principais informações a respeito dos instrumentos psicológicos, mais especialmente no que se refere à editora, data de publicação, variável medida, padronização, validade e precisão.

\section{Método}

\section{Instrumentos consultados}

Foram consultados 142 instrumentos psicológicos, originalmente comercializados pelas seguintes das editoras: CEPA, Vetor, Casa do Psicólogo, Edites, CETEPP, Mestre Jou, Editorial Psy, Manole, Artes Médicas, Edicon, Entreletras. Os nomes dos instrumentos e as respectivas referências encontram-se em anexo. descrição clara dos procedimentos de aplicação e correção (e) um manual contendo essas informações. Atualmente, os instrumentos comercializados estão em análise para se verificar quais atendem esses requisitos mínimos.

\section{Material}

Para a realização da pesquisa, os autores se basearam no questionário proposto por Prieto e Muñiz (2000), que procurou, originalmente, avaliar os testes usados na Espanha. Eles têm como proposta a avaliação de vários aspectos teóricos e técnicos dos instrumentos, dentre eles: nome do teste, autor, editor, data da publicação, variável medida (classificação geral da variável que se pretende medir), áreas de aplicação, formato dos itens (resposta livre, respostas dicotômicas, eleição múltiplas, tipo Likert, adjetivos bipolares, outros), número de itens, validade de construto, qualidade de critério, validade de conteúdo, precisão (procedimentos utilizados: teste-reteste, formas 
paralelas, homogeneidade), suporte (administração oral, lápis e papel, manipulativo, informatizado, outros), correção (crivo, leitura ótica, realizado por aplicador, com a ajuda de experts, outros), transformação da pontuação (característica não aplicável ao instrumento, normatizada, não normatizada), escalas utilizadas (percentil, QI, T, outros), tempo de aplicação, documentação oferecida (manual, livros ou artigos complementares, disquetes, CD, outros) e bibliografia básica fornecida pelo teste.

No presente estudo, foram avaliados os seguintes aspectos: data original, editora de publicação no Brasil, variável, padronização brasileira (se existe), data da padronização brasileira, presença ou ausência de estudos relacionados à validade e à precisão. Em relação aos dois últimos elementos, foram considerados estudos de qualquer natureza, nacional ou estrangeiro.

\section{Procedimento}

Todos os instrumentos foram avaliados a partir das informações contidas em seus respectivos manuais e, posteriormente, analisados.

\section{Resultados}

Na Tabela 1, apresentamos a distribuição dos 146 instrumentos comercializados no Brasil pelas editoras que os publicam; três editoras (Casa do Psicólogo, CEPA e Vetor) são responsáveis por 83,6\% dos instrumentos comercializados. Na Tabela 2, são apresentadas as variáveis avaliadas pelos instrumentos. De todos os instrumentos analisados, 41,1\% avaliam inteligência e/ou aptidões, e 36,3\% avaliam características de personalidade.

\begin{tabular}{|l|l|l|}
\hline $\begin{array}{l}\text { Tabela 1. Número de instrumentos comercializados pelas diferentes editoras. } \\
\text { Editora }\end{array}$ & F & $\%$ \\
\hline CEPA & 51 & 34,9 \\
Vetor & 45 & 30,8 \\
\hline Casa do Psicólogo & 26 & 17,8 \\
\hline Edites & 7 & 4,8 \\
\hline CETEPP & 2 & 1,4 \\
Mestre Jou & 1 & 0,7 \\
\hline Editorial Psy & 4 & 2,7 \\
Manole & 1 & 0,7 \\
Artes Médicas & 2 & 1,4 \\
\hline Edicon & 1 & 0,7 \\
\hline Entreletras & 6 & 4,1 \\
Total & 146 & 100,0 \\
\hline
\end{tabular}

Tabela 2. Distribuição dos instrumentos pelas variáveis principais que eles avaliam.

\begin{tabular}{|l|l|l|}
\hline Variável avaliada & F & $\%$ \\
\hline Inteligência & 31 & 21,2 \\
\hline Personalidade & 53 & 36,3 \\
\hline Interesses profissionais / orientação profissional & 14 & 9,6 \\
\hline Aptidões / habilidades & 29 & 19,9 \\
\hline Alfabetização / leitura e escrita / dificuldades escolares & 15 & 10,3 \\
\hline Desenvolvimento & 1 & 0,7 \\
\hline Atitude & 2 & 1,4 \\
\hline Alterações neuropsicológicas & 1 & 0,7 \\
\hline Total & 146 & 100,0 \\
\hline
\end{tabular}

Na Tabela 3, são apresentadas as datas de publicação dos instrumentos consultados. Vale ressaltar que, no caso de instrumento estrangeiro, considerou-se a data da publicação do material original, ou seja, a da primeira edição. Considerando o exposto, faz-se pertinente observar a inexistência dessa informação nos manuais de 23,3\% dos instrumentos publicados. Dos instrumentos em que essa informação era disponível, 
pode-se observar três períodos mais proeminentes de construção, a saber: as décadas de 40, 70 e 90 .

Tabela 3. Distribuição dos instrumentos em razão da data original de publicação

\begin{tabular}{|l|l|l|l|}
\hline Data da publicação & $\mathrm{F}$ & $\%$ & $\%$ Acumulada \\
\hline Sem informação & 34 & 23,3 & 23,3 \\
\hline $1920-1929$ & 2 & 1,4 & 24,7 \\
\hline $1930-1939$ & 4 & 2,7 & 27,4 \\
\hline $1940-1949$ & 16 & 11,0 & 38,4 \\
\hline $1950-1959$ & 10 & 6,8 & 45,2 \\
\hline $1960-1969$ & 10 & 6,8 & 52,1 \\
\hline $1970-1979$ & 25 & 17,1 & 69,2 \\
\hline $1980-1989$ & 11 & 7,5 & 76,7 \\
\hline $1990-1999$ & 26 & 17,8 & 94,5 \\
\hline $2000-2002$ & 8 & 5,5 & 100,0 \\
\hline Total & 146 & 100,0 & \\
\hline
\end{tabular}

Quanto às características psicométricas, buscou-se verificar se os manuais relatavam os estudos referentes à precisão e à validade ainda que, no caso de instrumentos estrangeiros, realizados no país de origem. Em relação à padronização, avaliou-se a existência de estudos feitos no Brasil. A Tabela 4 apresenta os resultados para os diferentes tipos de instrumentos. Para cada tipo, a tabela apresenta o número de manuais que relatavam estudos de precisão (segunda coluna), de validade (terceira coluna) de padronização brasileira (quarta coluna), simultaneamente de precisão e de validade (quinta coluna) e simultaneamente de precisão, validade e padronização brasileira (sexta coluna). Nas últimas três linhas, são apresentados o total de instrumentos de cada uma das características anunciadas anteriormente. As porcentagens foram calculadas em relação ao total de instrumentos de cada tipo apresentado na última coluna (por exemplo, 23 dos 31 instrumentos correspondentes a 74,2\% dos instrumentos de avaliação da inteligência que relatam em seus manuais pelo menos um estudo de precisão).

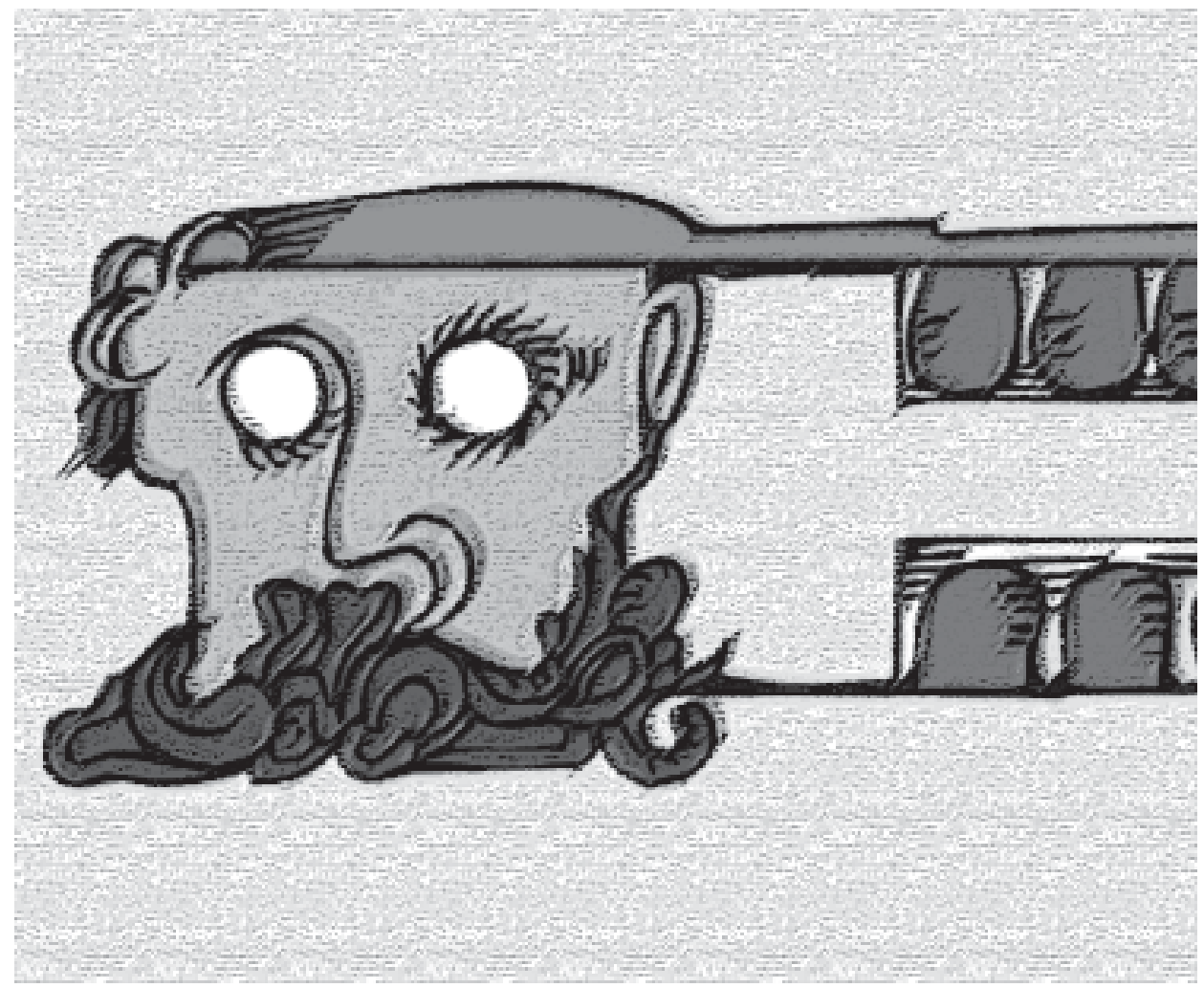




\begin{tabular}{|c|c|c|c|c|c|c|c|}
\hline Variável & & Precisão & Validade & Padroniz. & $\begin{array}{l}\text { Precisão } \\
\text { e Validade }\end{array}$ & $\begin{array}{l}\text { Precisão, } \\
\text { Validade, } \\
\text { Padroniz. }\end{array}$ & Total \\
\hline Inteligência & $\begin{array}{l}\mathrm{F} \\
\%\end{array}$ & $\begin{array}{l}23 \\
74,2\end{array}$ & $\begin{array}{l}23 \\
74,2\end{array}$ & $\begin{array}{l}16 \\
51,6\end{array}$ & $\begin{array}{l}20 \\
64,5\end{array}$ & $\begin{array}{l}13 \\
41,9\end{array}$ & $\begin{array}{l}31 \\
100\end{array}$ \\
\hline Personalidade & $\begin{array}{l}\mathrm{F} \\
\%\end{array}$ & $\begin{array}{l}17 \\
32,1\end{array}$ & $\begin{array}{l}19 \\
35,8\end{array}$ & $\begin{array}{l}20 \\
37,7\end{array}$ & $\begin{array}{l}14 \\
26,4\end{array}$ & $\begin{array}{l}11 \\
20,8\end{array}$ & $\begin{array}{l}53 \\
100\end{array}$ \\
\hline $\begin{array}{l}\text { Interesses profissionais / } \\
\text { orientação } \\
\text { profissional }\end{array}$ & $\begin{array}{l}\mathrm{F} \\
\%\end{array}$ & $\begin{array}{l}7 \\
50,0\end{array}$ & $\begin{array}{l}6 \\
42,9\end{array}$ & $\begin{array}{l}5 \\
35,7\end{array}$ & $\begin{array}{l}6 \\
42,9\end{array}$ & $\begin{array}{l}4 \\
28,6\end{array}$ & $\begin{array}{l}14 \\
100\end{array}$ \\
\hline Aptidões / habilidades & $\begin{array}{l}\mathrm{F} \\
\%\end{array}$ & $\begin{array}{l}17,0 \\
58,6\end{array}$ & $\begin{array}{l}19,0 \\
65,5\end{array}$ & $\begin{array}{l}18,0 \\
62,1\end{array}$ & $\begin{array}{l}15 \\
51,7\end{array}$ & $\begin{array}{l}11 \\
37,9\end{array}$ & $\begin{array}{l}29 \\
100\end{array}$ \\
\hline $\begin{array}{l}\text { Alfabetização } \\
\text { / leitura e escrita / } \\
\text { dificuldades escolares }\end{array}$ & $\begin{array}{l}\mathrm{F} \\
\%\end{array}$ & $\begin{array}{l}6 \\
40,0\end{array}$ & $\begin{array}{l}7 \\
46,7\end{array}$ & $\begin{array}{l}2 \\
13,3\end{array}$ & $\begin{array}{l}5 \\
33,3\end{array}$ & $\begin{array}{l}2 \\
13,3\end{array}$ & $\begin{array}{l}15 \\
100\end{array}$ \\
\hline Desenvolvimento & $\begin{array}{l}\mathrm{F} \\
\%\end{array}$ & 100 & $\begin{array}{l}1 \\
100\end{array}$ & 1 & & & $\begin{array}{l}1 \\
100\end{array}$ \\
\hline Atitude & $\begin{array}{l}\mathrm{F} \\
\%\end{array}$ & & $\begin{array}{l}1 \\
50\end{array}$ & $\begin{array}{l}1 \\
50\end{array}$ & & & $\begin{array}{l}2 \\
100\end{array}$ \\
\hline $\begin{array}{l}\text { Alterações } \\
\text { neuropsicológicas }\end{array}$ & $\begin{array}{l}\mathrm{F} \\
\%\end{array}$ & $\begin{array}{l}1 \\
100\end{array}$ & $\begin{array}{l}1 \\
100\end{array}$ & $\begin{array}{l}1 \\
100\end{array}$ & $\begin{array}{l}1 \\
100\end{array}$ & $\begin{array}{l}1 \\
100\end{array}$ & $\begin{array}{l}1 \\
100\end{array}$ \\
\hline Total & $\begin{array}{l}\mathrm{F} \\
\%\end{array}$ & $\begin{array}{l}71 \\
48,6\end{array}$ & $\begin{array}{l}77 \\
52,7\end{array}$ & $\begin{array}{l}64 \\
43,8\end{array}$ & $\begin{array}{l}61 \\
41,8\end{array}$ & $\begin{array}{l}42 \\
28,8\end{array}$ & $\begin{array}{l}146 \\
100\end{array}$ \\
\hline
\end{tabular}

Os dados revelaram que somente $28,8 \%$ apresentam todas as três características que são condições mínimas para se considerar um instrumento como cientificamente fundamentado. Obviamente, alguns instrumentos, principalmente os projetivos, não empregam dados normativos em seu sistema de interpretação, já que as variáveis avaliadas são qualitativas. Mesmo relaxando essa exigência e tomando somente os instrumentos que relatam simultaneamente estudos de precisão e de validade, observa-se que somente $41,8 \%$ apresentam essas características.

Deve-se, ainda, considerar que, relativamente aos parâmetros de precisão e validade, esta análise não discriminou se os estudos eram brasileiros, no caso de testes estrangeiros. Há que se considerar que, nesse caso, o número de instrumentos publicados com estudos de validade e precisão seria ainda menor.

A análise dessas propriedades nos instrumentos avaliados indica que os testes de personalidade são os que mais carecem dessas evidências e os de inteligência e aptidões são os que mais freqüentemente relatam estudos das propriedades básicas. Isso pode decorrer da maior facilidade em se pesquisar a validade de instrumentos de avaliação cognitiva em comparação com os instrumentos de avaliação da personalidade.

Tabela 5. Distribuição dos instrumentos em relação à data do último estudo de padronização brasileiro.

\begin{tabular}{|l|l|l|}
\hline Data & F & $\%$ \\
\hline $1935-1949$ & 6 & 4,1 \\
\hline $1950-1959$ & 3 & 2,1 \\
\hline $1960-1969$ & 7 & 4,8 \\
\hline $1970-1979$ & 20 & 13,7 \\
\hline $1980-1989$ & 19 & 13,0 \\
\hline $1990-1999$ & 51 & 34,9 \\
\hline $2000-2002$ & 12 & 8,2 \\
\hline Total com inf. & 118 & 80,8 \\
\hline Sem Informação & 28 & 19,2 \\
\hline Total & 146 & 100,0 \\
\hline
\end{tabular}

No que se refere à padronização, a Tabela 5 apresenta a data do último estudo brasileiro de padronização, dos 118 instrumentos que apresentaram esse tipo de estudo. Os resultados apontaram que 53,4\% dos instrumentos fizeram estudos mais recentes a partir da década de 90 . O 
restante apresenta informações mais antigas coletadas nas décadas de 70 e 80 .

\section{Discussão}

Os achados do presente estudo permitiram levantar uma série de implicações e reflexões que serão tratadas a seguir.

A constatação mais importante deste trabalho é que uma parte expressiva dos instrumentos comercializados carece de fundamentação científica e não atende aos requisitos mínimos definidos nas perspectivas nacionais ou internacionais, como, por exemplo, a Resolução 25/2001 (CFP, 2001) ou os padrões publicados pela American Educational Research Association, American Psychological Association, National Council on Measurement in Education. Em contrapartida, supõe-se que, se estudos de verificação de validade e precisão existem, além dos que foram comentados, eles não se encontram devidamente relatados nos respectivos manuais. Qual é a implicação desse fato?

O comportamento humano é complexo e sua avaliação também. Os instrumentos de avaliação propõem tarefas específicas às pessoas (resolver problemas, fazer um auto-relato, desenhar, contar histórias, dentre outros) como formas de se observar a manifestação de características psicológicas (traços latentes). A partir da maneira como as pessoas respondem, características psicológicas específicas podem ser inferidas. A associação entre manifestação-traço latente, no bojo da lógica dos instrumentos de avaliação, precisa ser desafiada e comprovada por meio de pesquisas empíricas, com a aplicação de métodos científicos adequados. As pesquisas, por sua vez, atestam a qualidade dos instrumentos. Se um instrumento não possui evidências de validade, não há segurança de que as interpretações sobre as características psicológicas das pessoas sugeridas pelas suas repostas sejam legítimas. Não se sabe o que o instrumento avalia realmente.

Outra característica ligada ao racional científico dos instrumentos é a precisão. Como nenhuma avaliação é livre de erro, deve-se buscar estimar o tamanho do erro esperado (ou erro padrão) cometido nas avaliações. Isso é feito por meio do estudo de precisão. Se um instrumento não possui evidências de precisão, não se tem idéia da magnitude do erro cometido nas avaliações, isto é, não se pode estimar quanto os resultados mudariam se fossem dadas novas oportunidades de avaliação, tais como outro profissional avaliando o mesmo candidato empregando o mesmo método, outro instrumento e/ou oportunidade com os mesmos objetivos.
Por último, há a questão da padronização. Em muitos instrumentos, a definição do que é esperado e, inversamente, do que é muito incomum ou diferente, é feita por comparação com grupos normativos. Isso auxilia a interpretação dos resultados obtidos. A inexistência de padronização brasileira ou a padronização inadequada no Brasil pode trazer dificuldades nas interpretações dos resultados, já que as pessoas serão comparadas com expectativas que podem ser inapropriadas. Por exemplo, pode existir um número grande de pessoas identificadas como mais deprimidas ou como apresentando dificuldades intelectuais; no entanto, essa proporção pode estar incorreta por causa do uso de expectativas inadequadas.

Considerando que muitos profissionais usam instrumentos sem que essas condições tenham sido estabelecidas em processos de seleção de pessoal, por exemplo, pode-se questionar sobre quantas decisões equivocadas os profissionais vêm tomando nesse contexto e qual a extensão desse prejuízo para a profissão e para a sociedade. De fato, na área de seleção, em especial, a avaliação psicológica tem-se tornado alvo de muitas críticas, principalmente no contexto de seleção.

Uma outra análise, ainda mais grave, é a de que muitos profissionais sequer pensam criticamente a respeito de tais questões ao utilizar um instrumento psicológico. De modo geral, esses resultados sugerem que a Psicologia brasileira tem-se caracterizado por despender pouca atenção aos fundamentos científicos de sua prática profissional, como também foi notado em alguns estudos avaliando o Provão de Psicologia (LandeiraFernanez, \& Primi, 2002, Pasquali, 2002).

Em síntese, a medida do CFP, no que se refere à avaliação dos testes comercializados, e a indicação, aos profissionais, dos que possuem condições mínimas, teve um grande impacto para reverter o problema aqui evidenciado. Na verdade, este trabalho permitiu retratar e, de alguma forma, justificar a necessidade de uma ação mais enfática no que diz respeito à situação da má qualidade dos instrumentos disponíveis aos profissionais de Psicologia, uma vez que apresentou o "estado da arte” da área de avaliação psicológica.

Ao contrário do que inicialmente podiam supor os psicólogos, esse movimento promoveu o crescimento da área, considerando que bons instrumentos e profissionais competentes e embasados por uma adequada formação profissional tendem a gerar ações mais qualificadas. Nesse sentido, uma formação profissional adequada na área de avaliação psicológica, se não resolver, pelo menos tende a minimizar algumas das dificuldades apontadas. Afinal, se a maioria 
dos profissionais tivesse um conhecimento básico de Psicometria, saberia julgar e distinguir instrumentos de boa e má qualidade e, possivelmente, não compraria instrumentos inadequados, inibindo sua comercialização pela baixa demanda.

Portanto, é preciso, paralelamente ao trabalho de avaliação dos instrumentos, tomar medidas para educar os profissionais e estudantes no que se refere aos princípios de Psicometria que sustentam os instrumentos e sua respectiva importância.

O presente estudo não pôde atender a todas as necessidades que a área requer e não era essa a pretensão. Aliado a isso, sugere-se que outros estudos sejam desenvolvidos, comparando os dados dos instrumentos antes-pós resolução bem como na direção da análise de outras variáveis ou ainda na análise das pesquisas de validade/precisão realizadas com amostras nacionais. 
Referências

ALCHIERI, J.C. \& BANDEIRA, D. R. Ensino de Avaliação Psicológicano Brasil. In R. Primi (org.). Temas em Avaliação Psicológica. Campinas: IDB Digital / Instituto Brasileiro de Avaliação Psicológica(IBAP),2002, pp.35-39.

ALVES, I. C. B.;ALCHIERI, J.C. \& MARQUES, K. Panorama Geral do Ensino das Técnicas do Exame Psicológico no Brasil. Anais do I Congresso de Psicologia Clínica. Universidade Presbiteriana Mackenzie. São Paulo, 2001, pp. 10-11.

ALVES FILHO, F. Qualquer uma das Alternativas: Conselho Federal de Psicologia Decide Avaliar, Pela Primeira Vez, a Eficácia dos Testes Psicológicos Usados no Brasil. Isto É, edição 1721, 25/09/2002, p. XXX.American Educational Research Association, American Psychological Association \& National Council on Measurement in Education. Standards for Educational and Psychological Testing. New York: American Educational Research Association, 1999.

ANASTASI, A. \& URBINA, S. Testagem Psicológica. Porto Alegre: Artes Médicas, 2000.

ANCONA-LOPEZ, M. (org.) Avaliação da Inteligência I. São Paulo: E.P.U., 1987.

AZEVEDO, M. M.; ALMEIDA, L. S.; PASQUALI, L. \& VEIGA, H. M. S Utilização dos Testes Psicológicos no Brasil: Dados de Estudo Preliminar em Brasilia. In L. S. Almeida; S. Araújo; M. M. Gonçalvez; C. Machado \& M. R. Simões (orgs.). Avaliação Psicológica: Formas e Contextos, vol. IV, Braga / APPORT, 1996, pp. 213-220.

BUTCHER, J. M. Revising Psychological Tests; Lessons Learned from the Revision of the MMPI. Psychological Assessment, 12(3), 2000, pp.263-271.

CFP - Conselho Federal de Psicologia. I Fórum Nacional de Avaliação Psicológica - Propostas Encaminhadas Para os Conselhos Federal e Regionais de Psicologia. Brasília, 2000.

CFP - Conselho Federal de Psicologia (2001a). Resolução 25/2001 Disponível em www.pol.org.br, consulta feita em 28/12/2001.

CFP - Conselho Federal de Psicologia (2001b). Resolução 30/2001. Disponível em www.pol.org.br, consulta feita em 28/12/2001.

ERTHAL, T. C. Manual de Psicometria. Porto Alegre: Artes Médicas, 1987.

HUTZ, C. S. \& BANDEIRA, D. R. Tendências Contemporâneas no Uso de Testes: uma Análise da Literatura Brasileira e Internacional. Psicologia: Reflexão e Crítica, 6(1/2),1993, pp. 85-101.

ITC-Comisión Internacional de Tests (2001). Diretrices Internacionales Para el Uso de los Tests. Colégio Oficial de Psicólogos. http:// www.cop.es/tests/Directrices.htm setembro 2001.

JACQUEMIN, A. Ensino e Pesquisa Sobre Testes Psicológicos. Boletim de Psicologia, v. XLV, n. 102, 1995, pp. 19-21.

KROEFF, P. Síntese de Posicionamentos a Serem Feitos Quanto ao Uso de Testes Psicológicos em Avaliação Psicológica. Anais da $18^{a}$ Reunião
LANDEIRA-FERNANEZ, J. \& PRIMI, R. Comparação do Desempenho Entre Calouros e Formandos no Provão de Psicologia 2000. Psicologia: Reflexão e Crítica. 15(1),2002, pp. 219-234.

LINK, N. L. H. Coding Thematic Apperception Test: Responses Using Rorschach Determinants Associated with Adlerian Definitions of Movement. Dissertation Abstracts International, 60 (6-B), 2950, 1999.

NORONHA, A. P. P. Análise de Coeficientes de Testes de Inteligência Publicados no Brasil. Psico, 32(2), pp.73-86.

NORONHA, A. P. P.; SARTORI, F. A.; FREITAS, F. A. \& OTTATI, F. Informações Contidas nos Manuais de Testes de Inteligência. Psicologia em Estudo, 6(2), 2001, pp. 101-106.

NORONHA, A. P. P.; SARTORI, F. A.; FREITAS, F. A. \& OTTATI, F. Informações Contidas nos Manuais de Testes de Personalidade. Psicologia em Estudo, 7(2), 2002, pp. 143-149.

NORONHA, A. P. P.; ALCHIERI, J. C. \& PRIMI, R. Elaboração de um Sistema de Referência Nacional Sobre Instrumentos de Avaliação Psicológica: Implantação de uma Base de Dados Relacional Sobre os Testes Psicológicos Comercializados no Brasil. Projeto de Pesquisa financiado pela FAPESP. Processo 2001/13.736-8, 2001.

PASQUALI, L. - editor. Instrumentos Psicológicos: Manual Prático de Elaboração. Brasília: LabPAM / IBAPP, 1999.

PASQUALI, L. O Provão de Psicologia ... In R. Primi (org.). Temas em Avaliação Psicológica (pp. ?-?). Campinas: IDB Digital / Instituto Brasileiro de Avaliação Psicológica (IBAP), 2002.

PASQUALI, L. \&ALCHIERI, J. C. Os Testes Psicológicos no Brasil. In L. Pasquali (org.). Técnicas de Exame Psicológico - TEP-Manual. São Paulo: Casa do Psicólogo, Conselho Federal de Psicologia, 2001, pp. 195-221.

PEREIRA,A. P. C. \& CARELLOS, S. D. M. S. Examinando o Ensino das Técnicas de Exame Psicológico. Cadernos de Psicologia, 3(4), 1995, pp.33-36.

PRIETO, G. \& MUÑIZ, J. 2000. Un Modelo Para Evaluar la Calidad de los tests utilizados en España. http://www.cop.es/tests/modelo.htm, $04 / 12 / 00$.

RIBEIRO, J. I. P. Experiências de Relações: seus Significados na Formação de Psicólogos. Psico, 27(2), 1996, pp. 23-47.

SILVERSTEIN, M. L.; MARSHALL, L. \& NELSON, L. D. Clinical and Research Implications of Revising Psychological Tests. Psychological Assessment, 12(3), 2000, pp. 298-303.

VASCONCELOS, Z. B. \& TOLEDO de SANTANA, C. M. O Ensino das Técnicas de Exame Psicológico na Universidade Federal da Paraíba. Anais do I Congresso de Psicologia Clínica. Universidade Presbiteriana Mackenzie, São Paulo, p.133. 
Allessandrini, C. D., Duarte, J. L. M., Dupas, M. A., \& Bianco, M. P. F. Teste do Desenho de Silver, São Paulo, Casa do Psicólogo, 1996.

Almeida, L. S., \& Primi, R. BPR-5, São Paulo: Casa do Psicólogo, 2000.

Alves, I. C. B., Duarte, J. L., \& Duarte, W. Escala de Maturidade Mental Columbia, São Paulo: Casa do Psicólogo, 1993.

Andrade, E. M., Moraes, R., \& Wendel, Z. S. Questionário de Personalidade Dadahie, Rio de Janeiro: CEPA, s/d.

Andrade, E. M., \& Alves, D. G. Dezesseis PF-Questionário 16 Fatores de Personalidade. Rio de Janeiro: CEPA, 1999, 5 a ed.

Angelini, A., Alves, I. C. B., Custódio, E. M., \& Duarte, W. M. Matrizes Progressivas Coloridas. São Paulo: Centro Editor de Testes e Pesquisas em Psicologia, 1999.

Antipoff, H. Teste Minhas Mãos-MM. Rio de Janeiro: CEPA, s/d.

Bastos, O. P. M. VIG Bateria Burocrática. São Paulo: Vetor, 1999.

Benczik, E. B. P. Escala de Transtorno de Déficit de Atenção/ Hiperatividade (versão para professores). São Paulo: Casa do Psicólogo, 2000.

Benkö, A. \& Simões, R. P. Inventário Multifásico de Personalidade de Minnesota-MMPI. Rio de Janeiro: CEPA, d/d.

Bessa, P. P. Teste do Catálogo de Livros Bessa-Tramer. Rio de Janeiro: CEPA, 1998.

Biaggio,A.M. B., \& Natalício, L. Inventário de Ansiedade Traço-Estado. Rio de Janeiro: CEPA, 1979.

Biaggio, A. Inventário de Expressão de Raiva como Estado e Traço STAXI. São Paulo: Vetor, 1992.

Biaggio,A., \& Natalício, L. Inventário de Ansiedade de Traço-Estado IDATE. São Paulo: Vetor, 1979.

Biaggio, A. Inventário de Ansiedade de Traço-Estado - IDATE C. Rio de Janeiro: CEPA, 1983.

Braga, G L. Bateria de Testes de Aptidão Geral. Rio de Janeiro: CEPA, 1993.

Brito, G. N. O. Escala de Avaliação do Comportamento Infantil Para o Professor. Rio de Janeiro: Entreletras, 1999.

Boccalandro, E. R. Atenção Concentrada AC-15. São Paulo: Vetor,1977. s/d. .Teste Verbal de Inteligência V-47. São Paulo: Vetor, Vetor, 2002 Teste Não Verbal de Inteligência-G-36. São Paulo: Vetor, 2002. Teste Não Verbal de Inteligência-G-38. São Paulo:

Boehm, A. E. Teste de Conceitos Básicos de Boehm. Rio de Janeiro: CEPA, 1977.

Both, F. J. MTB- Série Both. São Paulo: Casa do Psicólogo, 1974.

Brito, G. N. O. Escala de Avaliação do Comportamento Infantil Para o Professor- EACI-P. Rio de Janeiro: Entreletras, 1999.

Chamat, L. S. J. Coleção Papel de Carta. São Paulo: Vetor, 1997.

Cambraia, S. V. Atenção Concentrada. São Paulo: Vetor, 2002.
Campos, M. R. Teste de Maturidade para a Leitura. Rio de Janeiro: CEPA, 1994.

Campos, C. A. I., Duarte, M. R., \& Lima, T. Os Contos de Fadas e a Psicopedagogia. São Paulo: Vetor, 1998.

Campos, F. Matrizes Progressivas - Escala Geral. Rio de Janeiro: CEPA, 2001.

\section{$\overline{\text { CEPA, } 2002 .}$}

Matrizes Progressivas - Escala Avançada. Rio de Janeiro:

Catell, R. B., \& Catell, A. K. S. Teste Equicultural de Inteligência. Rio de Janeiro: CEPA, s/d.

Carvalhaes, J. Destreza Digital-TC. São Paulo: Vetor, s/d.

Clawson, A. Bender Infantil. Porto Alegre: Artes Médicas, 1980.

Costa, F. R. Teste de Raciocínio Lógico-Numérico. São Paulo: Vetor, s/ d.

1997. Escala de Personalidade de Comrey. São Paulo:Vetor,

. Teste de Atenção Concentrada, Rapidez e Exatidão. São Paulo: Vetor, 1999.

Corona, L. C. G. Escala Reduzida do Autoconceito ERA. Rio de Janeiro: CEPA, 1999.

. Escala de Preconceito Profissional-EPP. Rio de Janeiro: CEPA, s/d.

Cunha, J. Cornel Index - CI. Rio de Janeiro: CEPA, 1949.

Cunha, J., \& Nunes, M. L. T. Testes das Fábulas. São Paulo: Casa do Psicólogo, 2000.

Cunha, J. A. Escalas de Beck - Manual da Versão em Português. São Paulo: Casa do Psicólogo, 2001.

Cunha, J. A., Moraes, M. I. B., Rocha, N. S., Werba, L., Vale, R. R.,

Spader, M., Moraes, J. E. B. C., Salerno, I. C. \& Costa, L. Sondagem Intelectual. Rio de Janeiro: CEPA, s/d.

Cunha, S. E. Teste dos Conjuntos Emparelhados. Rio de Janeiro: CEPA, 1980.

Cunha, S. E.; Coutinho, M. T. C. (1993). Teste Diagnóstico de Habilidades do Pré-Escolar. Rio de Janeiro: CEPA, 1993, $2^{\mathrm{a}}$ ed.

Del Nero, C. Programa de Hábitos e Desempenho no Estudo - PHD. São Paulo: Vetor, 1997.

\section{2.}

. Teste de Capacidade Intelectual-TCI. São Paulo: Vetor,

Del Nero. Levantamento de Interesses Profissionais - LIP. São Paulo: Vetor, 1984.

Del Nero, C. Programa de Atitudes dos Pais - PAP. São Paulo: Vetor, 1997.

Vetor, s/d.

_Registro de Atitudes e Comportamento. São Paulo:

Del Prette, Z. A. P., \& Del Prette A. Inventário de Habilidades Sociais IHS. São Paulo: Casa do Psicólogo, 2001.

Devoet, B., \& Grossuin, P. Inventário e Auto-Análise dos Interesses Profissionais, Rio de Janeiro: CEPA, 2001.
Relação de Instrumentos 
Duarte, J. L. Teste D70. São Paulo: Centro Editor de Testes e Pesquisas em Psicologia, 1996

Edites. MacQuarrie Teste de Relações Espaciais. São Paulo: Edites, 1976.

Ferreira, M. C. Teste Evolutivo de Percepção Visual. Rio de Janeiro: Entreletras, 2001.

Figueiredo, V. Escala de Inteligência Weschler para Crianças III edição - WISC III. São Paulo: Casa do Psicólogo, 2002.

Freitas, A. M. L. Teste de Zulliger. São Paulo: Casa do Psicólogo, 1996. Freitas JR., O. Reprodução de Figuras. Rio de Janeiro: CEPA, 2001.

Goulart,I. B. Teste Raven de Operações Lógicas. Rio de Janeiro: CEPA, 1975.

Goulart, I. B., \& Bregunci, M. G. C. Teste de Aptidão Mecânica. Rio de Janeiro: Entreletras, 1998.

Gouveia, J. P., Ramalheira, C., Robalo, M. T., Borges, J. C., \& Almeida, L. R. Inventários de Expectativas e Crenças Pessoais Acerca do Álcool. São Paulo: Casa do Psicólogo, 1996.

Grados, J., \& Fernandéz, E. Teste de Frases Incompletas com Aplicação à Indústria-FIGS. Rio de Janeiro: Entreletras, 2000.

Growald, L., \& Growald, A. Teste de Compreensão Técnico-Mecânica. São Paulo: Vetor, 1983.

Guerra, D. N. Teste de Retenção Visual. Editora CEPA, 1995.

Heiss, R. O Teste das Pirâmides de Cores. São Paulo: Vetor, 1982.

Hutz, C. S., \& Nunes, C. H. S. S. Escala Fatorial de Ajustamento Emocional Neuroticismo. São Paulo: Casa do Psicólogo, 2001.

Justo, H., \& Van Kolck, T. O Teste das Pirâmides de Cores. São Paulo: Vetor, 1996.

Krausz, R. R. Diagnóstico Organizacional Forma I e Forma II. São Paulo: Casa do Psicólogo, 1994.

Inventário de Administração do Tempo ADT. São Paulo: Casa do Psicólogo, 1994.

Kuder, G. F. Kuder- Inventário de Interesses. Rio de Janeiro: Cepa, 2000.

Lipp, M. Inventário de Sintomas de Stress para Adultos de Lipp ISSL. São Paulo, Casa do Psicólogo 1994.

Lipp, M. E., \& Lucarelli, M. D. M. Escala de Stress Infantil ESI. São Paulo: Casa do Psicólogo, 1998.

Lopes, M. S., \& Freitas, A. M. L. Inventários da Atitude de Trabalho IAT. São Paulo: Casa do Psicólogo, 1997.

López, E. M. Questionário Íntimo. São Paulo: Vetor, s/d.

Maia, J. Prova de Nível Mental. Rio de Janeiro: CEPA, 1949.
Macquarrie, T. W. Teste de Relações Espaciais. São Paulo: Editora Edites, 1953.

Minicucci, A. Liderança e Poder. São Paulo: Vetor, 1986. . Teste Caracterológico-TCO. São Paulo: Vetor, 1996. . Teste Palográfico. São Paulo: Vetor, 1991.

1983. Teste de Estruturas Vocacionais-TEV. São Paulo: Vetor,

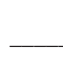
. Teste de Liderança Situacional-TLS. São Paulo: Vetor, 2002.

Mira y Lopez, E. Psicodiagnóstico Miocinético - PMK. Rio de Janeiro: CEPA, 2001.

Moraes, R., Andrade, E. M., \&Alves, D. G. Teste Coletivo de Inteligência Para Adultos. Rio de Janeiro: CEPA, 2001.

Murray, H. A., \& Morgan, R. Teste de Apercepção Temática TAT. São Paulo: Casa do Psicólogo, 1943.

Nagelschmidt, A. M. C. Mandalas de Palavras. São Paulo: Vetor, 1995.

Neiva, K. M. C. Escala de Maturidade Para a Escolha Profissional. São Paulo: Vetor, 1999.

Nero, C. D. Levantamento de Interesses Profissionais. São Paulo: Vetor, 1975 .

Programação de Hábitos e Desempenho no Estudo. São Paulo: Vetor, 1977.

. Panorama de Atitudes dos Pais. São Paulo: Vetor, 1997. Nijamkin, G. C., Braude, M. G., \& Tardico L. S. L. P. Questionário Desiderativo. São Paulo: Vetor, s/d.

Nick, E. Como Chefiar? Rio de Janeiro: CEPA, s/d. 1999. Inventário Ilustrado de Interesses GEIST. Rio de Janeiro: CEPA, . Dominós D-48. Rio de Janeiro: CEPA, 1999.

Novaes, M. H. Testes de Organização Percepto-motora. Rio de Janeiro: CEPA, 1980.

Oliveira, R. Questionário Vocacional de Interesse. São Paulo: Vetor, 1982.

Questionário do Adolescente. São Paulo: Vetor, 1988. . Avaliação de Chefia. São Paulo: Vetor, 1994.

. Teste Não Verbal de Inteligência Para Crianças-R2. São Paulo: Vetor, 2000. 
. Teste Não Verbal de Inteligência-R1. São Paulo: Vetor,

2000.

Oliveira, M. S. Figuras Complexas de Rey: Teste de Cópia e Reprodução de Memória de Figuras Geométricas Complexas. São Paulo: Casa do Psicólogo, 1999.

Pacheco, M. T. A. Teste de Apercepção Infantil CAT-S. Campinas: Editora Editorial Psy, 1992.

Teste de Apercepção Para Idosos. Editorial Psy,

1992.

Pleno, 2000. . Teste de Apercepção Infantil. São Paulo: Livro

Teste de Apercepção Infantil CAT-H. São Paulo:

Livro Pleno, 2000.

Pasquali, L., Gouveia, V. V., Andriola, W. B., Miranda, F. J. \& Ramos, A.L. M. Questionário de Saúde Geral de Goldberg QSG. São Paulo: Casa do Psicólogo, 1996.

Pasquali, L., Azevedo, M. M., \& Ghesti, I. Inventário Fatorial de Personalidade IFP. São Paulo: Casa do Psicólogo, 1997.

Pinto, E. B., Vilanova, L. C. P. \& Vieira, R. M. O Desenvolvimento do Comportamento da Criança no $1^{\circ}$ Ano de Vida. São Paulo: Casa do Psicólogo, 1997.

Piovani, C. M., Piovani, G. Teste Edites de Inteligência. São Paulo: Edites, s/d.

Piovani, G. Medida de Fluência Verbal. São Paulo: Edites, s/d. Memória-R. São Paulo: Edites, s/d.

Piovani, C. Bateria TSP. São Paulo: Edites, s/d.

Piovani, G., \& Piovani, C. Atenção Difusa-MPM. São Paulo: Edites, 1976.

Poppovic, A. M. \& Moraes, G. G. Prontidão Para a Alfabetização. São Paulo: Vetor, s/d.

Poppovic, A. M. Teste Metropolitano de Prontidão Forma R, São Paulo, Vetor, s/d.

Poppovic, A. M. Escala de Inteligência Wechsler Para Crianças - WISC. Rio de Janeiro: CEPA, 1964.

Rainho, O. Teste de Compreensão Mecânica Forma A. Rio de Janeiro: CEPA, 1978.

Rainho, O. \& Ribeiro, C. C. Cubos de Kohs. Rio de Janeiro: CEPA, 1993.

Rainho, O. Bateria Fatorial Cepa, Rio de Janeiro. Rio de Janeiro: CEPA, 2002.

Reale, D. O. Escala de Sociabilidade e Emotividade. São Paulo: Edites, s/d.

Rorschch, H. Teste de Rorschach. São Paulo: Editora Manole, 1921.
Schoenfeldt, B. K. Becasse Maturidade Escolar. São Paulo: Vetor, s/d. Souza, F. V., \& Cambrais, S. V. Teste dos Relógios. São Paulo: Vetor, 2002.

Stein, L. M. Teste de Desempenho Escolar TDE. São Paulo: Casa do Psicólogo, 1994

Tardivo, L. S. L. P. C. O Teste de Apercepção Infantil e o Teste das Fábulas de Duss. São Paulo: Vetor, 1998.

Thurstone, L. L., Angelini, A. L., \& Angelini, H. R. C. Inventário de Interesses Profissionais. Rio de Janeiro: CEPA, 2001.

Tonglet, E. C. Teste de Prontidão Emocional Para Motoristas. São Paulo: Vetor, 1998.

Bateria de Funções Mentais Para Motoristas - 1. São Paulo: Vetor, 1999

Bateria de Funções Mentais Para Motoristas - 2. São Paulo: Vetor, 2000.

Bateria de Funções Mentais Para Motoristas - 3. São Paulo: Vetor, 1999.

Bateria de Funções Mentais Para Motoristas 4. São Paulo: Vetor, 2002

Torroela, G. Questionário Confidencial. São Paulo: Vetor, s/d.

Vaz, C. E. Z-Teste Técnica de Zulliger. São Paulo: Casa do Psicólogo, 1998.

Villas-Boas, J.T. F. Teste Projetivo Omega. Rio de Janeiro, CEPA, s/d.

Wartegg, E. Teste de Completamento de Desenhos Wartegg. São Paulo: Centro Editor de Teste e Pesquisas em Psicologia, 1939.

Wechsler, S. M. Desenho da Figura Humana de Wechsler. Campinas: Editorial Psy, 1996.

Avaliação da Criatividade por Figuras e Palavras. Campinas: Impressão Digital do Brasil Gráfica e Editora Ltda, 2002.

Wechsler, D. WISC-III: Escala de Inteligência Weschsler Para Crianças. São Paulo: Casa do Psicólogo, 1991.

Weil, P., \& Nick, E. INV. Rio de Janeiro: CEPA, 1951

Witter, G.P., \& Copit, M. S. Lendo e Escrevendo. São Paulo: Vetor, s/

Zacharias, J. J. M. Questionário de Avaliação Tipológica-QUATI. São Paulo: Vetor, 2000

Zacharias, J. J. M., \& Prado, A. C. A. L. Diagnóstico Tipológico Organizacional. São Paulo: Vetor, s/d. 